Marcos Cantele

marcos.cantele@gmail.com

Especilialista em Gestão

de materiais e logística.

Universidade de Caxias do Sul.

Caxias do Sul, RS. BR.

Daniel Hank Miri

danielmirid@gmail.com

Mestrando em Administração

pela Universidade de Caxias do

Sul. Caxias do Sul, RS. BR.

Zaida Cristine dos Reis

zcreis@gmail.com

Doutora em Administração.

Professora da Universidade de

Caxias do Sul. Caxias do Sul, RS. BR.

Vanessa de Campos Machado machadv@miamioh.edu

Mestra em Administração.

Professora doInstituto Federal

de Educação, Ciência e

Tecnologia do Rio Grande do

Sul. Porto Alegre, $\mathrm{RS}, \mathrm{Br}$.

Paula Patrícia Ganzer ganzer.paula@gmail.com Pós-Doutora em Administração. Coordenadora dos Cursos de Graduação em Administração,

Tecnologia em Processos

Gerenciais e Tecnologia em

Gestão de Recursos Humanos na

Faculdade CNEC Farroupilha.

Farroupilha, RS, BR.

\section{Cassiane Chais}

cassichais@gmail.com

Doutora em Administração.

Bolsista da APEX-Brasil e

Universidade de Passo Fundo,

atuando como técnica em

exportação pelo Programa PEIEX.

Juliana Matte

ju.cxs1@gmail.com

Doutoranda em Administração

pela Universidade de Caxias do

Sul. Caxias do Sul, RS. BR.

Pelayo Munhoz Olea pelayo.olea@gmail.com Pós-Doutor em Gestão Ambiental. Universidade Federal do Rio Grande. Rio Grande, RS, BR.

\author{
ARTIGOS
}

\section{GESTÃO DOS PRODUTOS COM BAIXO GIRO DE ESTOQUE EM UMA INDÚSTRIA DE MÉDIO PORTE DO SETOR ELÉTRICO}

\author{
MANAGEMENT OF PRODUCTS WITH LOW \\ INVENTORY TURNOVER IN A MID-SIZE \\ INDUSTRY OF THE ELECTRICAL SECTOR
}

\section{RESUMO}

O objetivo deste artigo consiste em identificar as causas da obsolescência dos produtos em uma empresa de médio porte do setor elétrico, cujo processo de fabricação é caracterizado pelo Engineering-to-Order(ETO), propondo ações para sanar tal problemática. Para isso, procedeu-se à aplicação de entrevistas semiestruturadas e à coleta de documentos e relatórios da empresa em estudo. Foram identificados como causas solicitação de compra de materiais para projetos especiais, troca de itens de engenharia sem revisar o estoque, cadastro de produtos descentralizados, alteração do projeto com troca de material, compra de materiais por meio de kits e ausência de método que informe a existência de itens similares. Em face disso, asações propostas foram gerar pré-estrutura para compra conforme necessidades, realizar análise de estoques e compras para cadastro de novos produtos, limitar o número de usuários para cadastro de matéria-prima, enviar ao cliente materiais que sobraram em decorrência de alteração de projeto e utilizar os itens de forma avulsa.

Palavras-chave: Gestão de Estoques. Obsolescência de Produtos. Baixo Giro de Produtos.

\begin{abstract}
The objective of this article is to identify the causes of product obsolescence in a medium-sized company in the electrical sector, whose manufacturing process is characterized by Engineering-to-Order (ETO), proposing actions to remedy this problem. To this end, semi-structured interviews were applied and documents and reports of the company under studywere collected. The following causes were identified: request for purchase of materials for special projects, exchange of engineering items without reviewing the stock, registration of decentralized products, changing of the
\end{abstract}


project with exchange of material, purchase of materials through kits and absence of a method to inform the existence of similar items. Therefore, the proposed actions were to generate pre-structure for purchase according to needs, to perform stock analysis and purchases for registration of new products, to limit the number of users for raw material registration, to send the client materials left over as a result of project change and to use the items in a separate way.

Keywords: Inventory Management. Product Obsolescence. Low Product Turnovers.

\section{INTRODUÇÃO}

Para suprir determinadas irregularidades na cadeia de fornecimento, uma das estratégias adotadas pelas empresas consiste em ter alguns itens com estoque de segurança. Trata-se, assim, de manter níveis de estoque suficientes para evitar faltas de estoque perante a variabilidade da demanda e da incerteza do ressuprimento do produto quando necessário (WANKE, 2011).

Os produtos usados em uma empresa passam pelo sistema em uma sequência, e uma execução completa de todos os produtos forma um ciclo de produção. Nesse sentido, as organizações contam com um modelo integrado de produção, estoque e manutenção preventiva, caracterizado pelo conceito de tempo de atraso, e seu sistema de produção, geralmente, produz vários itens sequencialmente com a manutenção preventiva em alguns momentos de configuração - como observado na indústria (LIU; WANG; PENG, 2015). Ressalta-se, contudo, que a produtividade atual e a utilização de equipamentos, normalmente, são baixas na indústria manufatureira e que a atual manipulação de distúrbios de produção nessa indústria não é eficaz (YLIPÄÄ et al., 2017).

Modelos de trabalho de alto envolvimento estão associados a altos níveis de influência dos trabalhadores sobre o processo de trabalho, a exemplo de altos níveis de controle sobre como realizar tarefas ou de altos níveis de en- volvimento na elaboração de procedimentos de trabalho. Assim, o nível de interdependência dentro de um sistema de produção tem implicações importantes para o tipo de envolvimento dos funcionários que a gerência considerará útil. Quando a interdependência está em alto nível em um sistema de produção, é um erro imaginar que a administração estará pensando no projeto de trabalho, principalmente no nível do trabalho individual (BOXALL; WINTERTON, 2018).

No Engineering-to-Order (ETO), o estilo de alta variedade dos negócios e o uso de sistemas Enterprise Resource Planning (ERP) afetam a capacidade de implementação tecnológica eficiente (CARNIEL-PERRIN et al., 2016). Os sistemas não padronizados devem, portanto, ser projetados para atender aos requisitos do cliente, evitando, desse modo, a manutenção de estoques (SYLLA et al., 2018).

Tendo isso em vista, o objetivo deste estudo consiste em identificar as causas da obsolescência dos produtos em uma empresa de médio porte do setor elétrico, cujo processo de fabricação é caracterizado pelo ETO, propondo ações para sanar tal problemática. A fim de alcançar tal objetivo, este artigo está estruturado em quatro seções, além desta introdução. A seguir, apresentam-se o referencial teórico sobre gestão de estoques e obsolescência dos produtos, os procedimentos metodológicos adotados para a realização da pesquisa, os resultados obtidos e as considerações finais.

\section{REVISÃO DA LITERATURA}

\subsection{GESTÃO DE ESTOQUES}

O efeito de desempenho da cadeia de suprimentos varia de acordo com o sistema de produção. Enquanto a integração dessa cadeia com o cliente melhora o desempenho de produção única, a integração com os fornecedores melhora o desempenho da produção em lote. A integração com fornecedores apenas contribui para o desempenho de custo da produção em massa (SHOU et al., 2018), e 
não para o desempenho de sistemas de alta disponibilidade, em que peças sobressalentes de alto custo, frequentemente, seguem padrões de demanda irregulares, com valores de demanda zero (COSTANTINO et al., 2018).

Ressalta-se, ainda, que, por meio de um modelo de inventário dinâmico que capta, explicitamente, as decisões periódicas de uma empresa sobre a quantidade de reabastecimento de estoques, o montante de dividendos líquidos de subscrições de capital e o valor da dívida em curto prazo, demonstrou-se que, tendo em vista decisões operacionais e financeiras de curto prazo coordenadas, o nível ótimo de estoque aumenta, inicialmente, à medida que sua dívida em longo prazo aumenta e que, depois que a empresa esgota sua capacidade de endividamento em curto prazo, à medida que a dívida em longo prazo aumenta ainda mais, o nível de estoques diminui e depois permanece constante (HU, 2017). Além disso, sabe-se que os registros de inventário de matérias-primas, de produtos em processo e de produtos finais no fabricante e nos varejistas são imprecisos, já que as matérias-primas podem ser ordenadas por uma subpolítica de reabastecimento de acordo com o consumo previsto de matérias-primas no processo de produção. Diante disso, a partir de experimentos numéricos, verificou-se que, além de implantar dispositivos de identificação por radiofrequência (RFID) em sistemas de gerenciamento de estoque de matérias-primas (WIPs) e de produtos finais do fabricante e dos varejistas, políticas robustas e integradas podem ser empregadas para proteger a organização contra o impacto da imprecisão do estoque (LI; WANG, 2018).

Destaca-se, também, que, como os custos de estoque representam metade dos custos de logística, o gerenciamento ideal de estoque para minimizar os custos totais de estoque continua sendo uma vantagem competitiva sustentável. Nesse cenário, o transbordo lateral constitui, evidentemente, uma estratégia comprovada para minimizar os custos totais de estoque, já que os ônus adicionais são mais do que compensados pela redução dos custos de falta de estoque (NAKANDALA; LAU; SHUM, 2017). Na prática, observa-se que uma distribuição precisa da demanda é, muitas vezes, distorcida para muitos itens e que a distribuição normal à demanda aleatória pode causar uma grande perda financeira para um sistema de estoque/produção (GHOLAMI; MIRZAZADEH, 2018).

Assim, o gerenciamento de estoques nos complexos ambientes de manufatura atuais tornou-se, cada vez mais, desafiador. Como o gerenciamento ineficiente de estoques pode ocasionar escassez de material, estoques excessivos, prazos de entrega longos, perda de espaço e falta de serviço ao cliente, atualmente, várias empresas estão usando sistemas de informações para estabelecer vínculos efetivos com fornecedores, clientes e outros agentes da cadeia de suprimentos (AQLAN, 2017). Parece relevante para a prática de gerenciamento de estoque permitir a incerteza em momentos de possíveis alterações, a fim de modelar mudanças de demanda que ocorrem por razões não observadas (WANG; MERSEREAU, 2017).

Nesse contexto, a otimização conjunta das decisões de alocação de capacidade, estoque e demanda tem significativa redução de custos em uma decisão sequencial e leva a uma alta utilização da unidade de produção no que concerne aos custos de capacidade linear, mas a uma utilização relativamente baixa no que concerne aos custos côncavos. O estoque de segurança, por sua vez, implica custos lineares e côncavos relativamente baixos nos centros de distribuição. E as decisões de alocação de demanda têm economias de custos significativos em comparação a uma decisão sequencial e levam a uma alta utilização da instalação de produção no que se refere a custos de capacidade linear, mas há uma utilização relativamente baixa no que se refere a custos côncavos (BAYRAM; GZARA; ELHEDHLI, 2019).

\subsection{OBSOLESCÊNCIA DOS PRODUTOS}

A obsolescência ocorre quando os ele- 
mentos do sistema se tornam desatualizados. Essa situação provoca implicações operacionais, logísticas, de confiabilidade e de custo (BURNS, 2016).

Os produtos que se tornam obsoletos causam aumento de custos para as organizações devido à interrupção do fluxo normal de negócios. Essas interrupções podem ser vistas na falta, no empilhamento e na reformulação forçada de produtos e componentes. Para minimizar o impacto da obsolescência do produto, as empresas devem integrar os métodos de previsão nos processos de negócios a fim de permitir um gerenciamento proativo, que faça que as organizações maximizem o tempo para tomar uma decisão e aumentem o número de decisões viáveis (JENNINGS, 2018).

O momento e a razão da obsolescência de um produto são de particular importância para a satisfação do cliente e a decisão de recompra. As razões para a obsolescência são múltiplas e, geralmente, desconhecidas para a empresa produtora. Além das falhas do produto, diminuição no design moderno, mudanças nas necessidades do cliente e novas tecnologias disponíveis podem causar obsolescência (ADETUNJI; BISCHOFF; WILLY, 2018).

Essa obsolescência pode tomar a forma de inoperabilidade funcional de um produto (obsolescência tecnológica ou funcional), de propostas desfavoráveis de custo-benefício para prolongar a vida útil do produto (obsolescência econômica) ou de valor declinante de produtos como moeda social e simbólica (obsolescência simbólica ou psicológica). Quanto ao primeiro tipo de obsolescência, ressalta-se que os produtos centrados em tecnologia, geralmente, contêm peças, softwares e materiais que duram menos que o ciclo de vida do próprio produto. Em relação ao segundo tipo, sabe-se que desvios de ciclo de vida entre peças e produtos podem resultar em grandes custos de ciclo de vida para produtos essenciais de missão, segurança e infraestrutura (SANDBORN, 2017). E, por fim, no que diz respeito ao terceiro tipo, essa forma de obsolescência pode ser estimulada por uma desestabilização, conduzi- da pelo fabricante, de fatores e características que qualificam os produtos como atraentes e funcionais (ECHEGARAY, 2016).

\section{METODOLOGIA}

Esta pesquisa adotou o método do estudo de caso único. O processo lógico para o desenvolvimento do estudo de caso único consiste no quanto ele é representativo para confirmar, contestar, testar ou estender a teoria e também satisfazer todas as condições para testá-la. Assim, a essência de um estudo de caso reside emtentar esclarecer uma decisão ou um conjunto de decisões e o motivo pelo qual foram tomadas, como foram implementadas e que resultados geraram (YIN, 2015).

Apesar de a estratégia de pesquisa empregada ser essencialmente qualitativa, foram usadas, ao mesmo tempo, técnicas qualitativas e quantitativas. Tais abordagens não são excludentes, uma vez que complementam a análise da realidade e a dinâmica do fato observado. Nessa perspectiva, as técnicas qualitativas e quantitativas deixam de ser opostas, para serem entendidas como complementares (TEIXEIRA; PACHECO, 2005).

O ambiente de pesquisa é uma empresa do segmento elétrico, cujo sistema de produção é classificado como ETO - EngineeringToOrder, ou seja, fabricação por encomenda com desenvolvimento de engenharia. O fluxo de aquisições e fabricação é dado após a liberação da engenharia de produto, fazendo que sejam comprados somente os produtos e as quantidades necessárias a serem utilizadas em determinado projeto. Por isso, a administração de materiais é complexa, pois é necessário sincronizar a data de entrega do projeto com a data de entrega das matérias-primas para o processo posterior, isto é, a montagem propriamente dita. Dessa forma, há interação entre a cadeia de abastecimento e o controle de estoques, que resulta em alto impacto na realização do projeto como um todo.

Os dados foram coletados por meio de documentos, registros em arquivos, entrevistas 
e observação participante. A técnica de entrevistas pessoais, com questionamentos verbais aos quatro principais envolvidos no processo de materiais da empresa selecionada para o estudo, foi utilizada durante o primeiro semestre de 2019. O roteiro de entrevista, adaptado do instrumento de pesquisa de Silva (2014), abordo um acrofluxo do processo, aquisições e estoques, obsolescência e sugestões para melhorias.

$\mathrm{Na}$ pesquisa documental, foram levantados documentos referentes à organização da estrutura logística da empresa, aos dados históricos da implantação dos estoques no sistema ERP eaos indicadores de desempenho que monitoram o índice de obsolescência em relação ao estoque total, ou seja, permitem identificar e quantificar o desempenho dos produtos obsoletos em relação ao total de matérias-primas estocadas (YIN, 2015).

Por meio da observação participante, obteve-se acesso às informações, que vão desde relatórios, documentos e manuais até o sistema de dados da empresa em estudo. A observação participante envolve uma interação social entre o pesquisador e o grupo, capacitando o observador a estudar em primeira mão a expe- riência do dia a dia e os procedimentos e, se necessário, interagir e discutir com a equipe sobre interpretações e situações particulares do fenômeno investigado. Além disso, na observação participante, o pesquisador deve ter mente aberta, tenacidade e determinação para adaptar-se aos diferentes tipos de pessoas e situações (CASSEL; SYMON, 1994).

\section{APRESENTAÇÃO E ANÁLISE DOS RESULTADOS}

O presente estudo de caso foi realizado em uma empresa de automação industrial de médio porte do setor elétrico, situada na região de Caxias do Sul. A produção dessa empresa se caracteriza como ETO. Assim, por meio de uma necessidade específica do cliente, realiza o levantamento de dados, as especificações, restrições e outros fatores essenciais para o desenvolvimento da solução ofertada. Somente após a aceitação da proposta comercial por parte do cliente, iniciam-se os processos internos da empresa.

A seguir, na figura1, apresenta-se o macrofluxo do desenvolvimento do projeto como um todo.

Figura 1 - Macrofluxo do processo de negócio

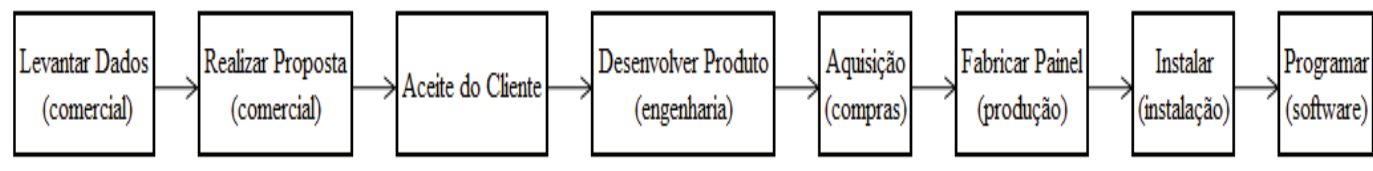

Fonte: elaborado pelos autores (2019).

Nota-se, na figura1, que o processo tem início na identificação da necessidade do cliente, etapa realizada pela equipe de vendas. Posteriormente, ainda na área comercial, ocorre o levantamento dos dados técnicos para a realização da proposta comercial, o que é feito pela equipe comercial com o apoio técnico da engenharia de desenvolvimento, quando necessário.

Quando o cliente aceita a proposta, insere-se um pedido no sistema ERP da empresa, o qual sinaliza chamados para os demais setores envolvidos no projeto. É nesse momento que a engenharia inicia o desenvolvimento do produto com base em um documento previamente preenchido pelo setor comercial e que os demais setores, como instalação e programação, são comunicados acerca de sua participação ou não no projeto.

Com o desenvolvimento efetuado pela engenharia, que inclui estrutura de produto, roteiro de fabricação, diagrama elétrico e diagrama mecânico, o pedido é liberado no ERP, e, com isso, as necessidades de materiais e recursos de fabricação são calculadas pela ferramen- 
ta de Material Requirement Planning(MRP),que o sistema disponibiliza. Somente após essa etapa, começa o processo de aquisição de materiais, baseado na estrutura de produto desenvolvida pela engenharia. Uma vez que os materiais integraram o estoque para determinado projeto, é feita a separação dos materiais por meio de ordens de produção (OPs), momento em que o Planejamento e o Controle da Produção (PCP) definem prioridades, conforme o cronograma do projeto.

O processo de fabricação resume-se em montagem com mão de obra humana, não havendo integração com máquinas de grande porte, somente uso de ferramentas manuais, como parafusadeiras e furadeiras. A montagem consiste em interpretar o diagrama elétrico e fazer as conexões elétricas de acordo com o indicado no plano elétrico. Finalizada essa etapa, o painel elétrico é entregue ao cliente.

A necessidade de instalar e de programar é definida conforme o tipo de solução ofertada ao cliente, de maneira que essa etapa pode ou não ser necessária. Tendo isso em vista, este estudo de caso limita-se a abarcar desde o início do processo até a fase de fabricação do painel elétrico nas dependências da empresa, de modo que etapas como programação e instalação não sejam contempladas.

\subsection{AQUISIÇÕES E ESTOQUES}

Conforme abordado anteriormente, para a empresa trabalhar com o sistema ETO, as matérias-primas necessárias a cada projeto somente são compradas após a liberação da engenharia de desenvolvimento e o cálculo do MRP. Assim, essa situação requer uma cadeia de fornecedores qualificada e comprometida com o resultado, pois o prazo de entrega para o cliente final será definido pelo status dos materiais comprados e pelo tempo de montagem (PESSOTI; SOUZA, 2005).

Além disso, alguns casos, que não necessariamente passam por todos os processos citados na seção anterior, merecem atenção especial em relação à compra de matéria-prima: a) solicitação por parte do setor comercial ao principal fornecedor de um orçamento para uma pré-lista de componentes (que, posteriormente, pode-se transformar na lista de compras) para melhor assertividade na proposta comercial;

b) compras estratégicas negociadas diretamente pela direção;

c) aumento das matérias-primas;

d) sinalização do cliente em fechar a compra do projeto e opção da empresa por comprar os materiais com uma pré-estrutura sem o desenvolvimento completo da engenharia.

As situações supracitadas ocorrem em função do compromisso com o cliente em relação ao prazo de entrega, pois os materiais, normalmente, são de origem estrangeira, o que impacta diretamente a data de entrega final (LI; WANG, 2018). Ressalta-se, ainda, que, para suprir algumas irregularidades na cadeia de fornecimento, a empresa também opta estrategicamente por ter alguns itens com estoque de segurança, o que, para Wanke (2011), constituiu ato de manter níveis de estoque suficientes para evitar faltas de estoque diante da variabilidade da demanda e da incerteza do ressuprimento do produto quando necessário.

\subsection{ANÁLISE CRÍTICA}

Atualmente, as demandas de compras são geradas por meio do ERP da empresa, o qual fornece inúmeras informações como valor, melhor fornecedor, data de entrega, quantidade e código do fornecedor. Por meio do MRP, a necessidade é gerada, e, então, o componente é comprado. Esse processo constituiu procedimento correto quando se trabalha sob projeto, pois, somente no momento em que a engenharia liberar o pedido, tem-se a certeza de que os produtos serão realmente utilizados. Uma das críticas ao processo de aquisição consiste justamente nessa falta de procedimento para alguns casos, em que o material é comprado por meio de uma pré-lista, não sendo necessaria- 
mente utilizado em sua totalidade (DE MAN; STRANDHAGEN, 2018).

Produtos com obsolescência programada por parte do fornecedor também são identificados como um ponto crítico. Por vezes, o fornecedor tem a necessidade de criar itens com uma tecnologia mais avançada, fazendo que o estoque contabilizado fique em desuso, pois a engenharia procura manter-se atualizada com a evolução dos produtos (BURNS, 2016). Outro ponto a ser abordado diz respeito às revisões de produtos criados pela própria empresa, ou seja, aos casos em que componentes de matéria-prima são atualizados em função de algumas mudanças no produto, criando-se novos códigos para manter o controle de estoque, o que faz que os itens desatualizados permaneçam em estoque até que fiquem obsoletos (HU, 2017).

Compras para garantir o desconto e os pacotes que o cliente sinaliza interesse em comprar também evidenciam pontos críticos do processo, pois não há certeza de o que está sendo comprado será utilizado realmente pela engenharia. Nessas situações, corre-se o risco de adquirir sem ter a certeza do consumo, o que é prática do negócio da empresa por querer garantir o preço e o prazo ao cliente (LIU; WANG; PENG, 2015).

$\mathrm{Na}$ gestão dos estoques, é feita a contagem cíclica diária de 50 itens para que, ao final do exercício fiscal, o estoque total tenha sido contado quatro vezes, eliminando, assim, a necessidade da contagem total. O estoque é medido pelo indicador de acuracidade, que também se mostra essencial para a gestão de estoques, a qual é controlada pela gestão industrial da empresa. A tabela 1 apresenta os resultados do indicador de acuracidade.

Tabela 1 - Indicador de acuracidade

\begin{tabular}{|c|c|c|c|c|c|}
\hline Períodos & Mês & Itens contados & Itens corretos & Resultado & Meta \\
\hline \multirow{3}{*}{$\begin{array}{c}1^{\circ} \\
\text { Trimestre }\end{array}$} & Janeiro & 298 & 283 & $94,97 \%$ & $95,00 \%$ \\
\hline & Fevereiro & 298 & 290 & $97,32 \%$ & $95,00 \%$ \\
\hline & Março & 100 & 95 & $95,00 \%$ & $95,00 \%$ \\
\hline \multirow{3}{*}{$\begin{array}{c}2^{o} \\
\text { Trimestre }\end{array}$} & Abril & 200 & 176 & $88,00 \%$ & $95,00 \%$ \\
\hline & Maio & 800 & 788 & $98,50 \%$ & $95,00 \%$ \\
\hline & Junho & 750 & 731 & $97,47 \%$ & $95,00 \%$ \\
\hline \multirow{3}{*}{$\begin{array}{c}3^{\circ} \\
\text { Trimestre }\end{array}$} & Julho & 800 & 767 & $95,88 \%$ & $95,00 \%$ \\
\hline & Agosto & 750 & 719 & $95,87 \%$ & $95,00 \%$ \\
\hline & Setembro & 950 & 937 & $98,63 \%$ & $95,00 \%$ \\
\hline \multirow{4}{*}{$\begin{array}{c}4^{\circ} \\
\text { Trimestre }\end{array}$} & Outubro & & & & $95,00 \%$ \\
\hline & Novembro & & & & $95,00 \%$ \\
\hline & Dezembro & & & & $95,00 \%$ \\
\hline & Acumulado & 4.946 & 4.786 & $96,77 \%$ & $95,00 \%$ \\
\hline
\end{tabular}

Fonte: elaborado pelos autores (2019).

Como o controle dos itens obsoletos e sua possível aplicabilidade passaram a ser de grande importância para a empresa, criou-se, então, o indicador de obsolescência de produtos, que tem como principal objetivo medir a evolução e o acompanhamento dos percentuais em relação ao estoque total (JENNINGS, 2018). Partindo desse princípio, adotam-se ações para a redução ou a eliminação dos produtos julgados fora de uso. Na figura3, é possível visualizar o acompanhamento das medições realizadas mensalmente.

$\mathrm{O}$ indicador é medido na forma de faixas de movimentação, com periodicidade mensal. Cada coluna representa uma faixa de medição, cujo resultado consiste em um percentual, cal- 
culado com base no somatório das faixas de 181 até 360 e da faixa de 361 dividida pelo total do estoque da empresa. Esse indicador também é apresentado nas reuniões de análise crítica da direção para tomada de decisão quanto ao destino dos materiais das faixas medidas. A seguir, na tabela 2 , constam os valores em reais e percentuais dos resultados do indicador de obsolescência.

Tabela 2 - Indicador de obsolescência

\begin{tabular}{|c|c|c|c|c|c|c|c|c|c|c|}
\hline & Mês & $<\operatorname{mov}<60$ & $61<\operatorname{mov}<90$ & $91<$ mov $<120$ & $121<$ mov $<180$ & $181<\mathrm{mov}<360$ & Mov $>361$ & Total R\$ & Resultado & Meta \\
\hline \multirow{3}{*}{$\begin{array}{c}1^{\circ} \\
\text { TRI }\end{array}$} & JAN & $1.327 .673,59$ & $49.173,47$ & $29.021,53$ & $69.156,86$ & 55.9 & $9.849,49$ & 2.089 & 3,1 & $4,00 \%$ \\
\hline & FEV & 1.210. & 26.2 & $28.964,14$ & 56. & 63 & 9,52 & 1.786. & & $4,00 \%$ \\
\hline & MAR & $729.703,30$ & $337.845,96$ & $17.735,93$ & 44. & & $26.842,14$ & $1.818 .665,88$ & $5,85 \%$ & $4,00 \%$ \\
\hline \multirow{3}{*}{$\begin{array}{c}2^{\circ} \\
\text { TRI }\end{array}$} & $\mathrm{ABR}$ & 674.6 & 129. & 266 & 24. & 75. & 3,54 & $1.874 .614,65$ & $5,90 \%$ & $4,00 \%$ \\
\hline & MAI & $746.487,80$ & $51.799,15$ & $111.512,58$ & $254.172,90$ & 81.8 & $54.738,45$ & $1.985 .803,37$ & $6,88 \%$ & $4,00 \%$ \\
\hline & JUN & 662.5 & 83. & & 284 & & 5,36 & 1.992 & $1 \%$ & $4,00 \%$ \\
\hline \multirow{3}{*}{$\begin{array}{c}3^{\circ} \\
\text { TRI }\end{array}$} & JL & 671.3 & 139. & 51. & & 232 & 2,25 & 1.888 & $15,44 \%$ & $4,00 \%$ \\
\hline & AGO & 2,14 & 81.4 & & & & 0,63 & 2.285 & $2 \%$ & $4,00 \%$ \\
\hline & SET & 1.27 & 67.2 & & & & 3,94 & 2.313 & & $4,00 \%$ \\
\hline \multirow{3}{*}{$\begin{array}{l}4^{\circ} \\
\text { TRI }\end{array}$} & OUT & 1.014 & 465.4 & 57.3 & $100.625,12$ & 181. & $25.366,36$ & 2.381 & $8,68 \%$ & $4,00 \%$ \\
\hline & NOV & & & & & & & & & $4,00 \%$ \\
\hline & DEZ & & & & & & & & & $4,00 \%$ \\
\hline \multicolumn{2}{|c|}{ Média } & $949.822,57$ & $143.124,69$ & $73.504,60$ & $107.317,45$ & $125.578,69$ & $31.591,67$ & 20416803,07 & $8,68 \%$ & $4,00 \%$ \\
\hline
\end{tabular}

Fonte: elaborado pelos autores (2019).

Pelo sistema de produção ETO, a empresa tem a consciência de que é aceitável estocar produtos por um período de até 180 dias, pois também leva em consideração o tempo de reposição de alguns itens que são importados. Para itens de prazo superior a 180 dias, deve-se estar alerta para tomar ações corretivas, tais como utilizar o produto em outro projeto, promover sua venda ou, em última ocasião, descartá-lo.

Com base na tabela 2, nota-se que é difícil controlar o indicador de obsolescência para que se mantenha dentro da meta. Nesse sentido, ressalta-se que a empresa entende que se trata de um ponto crítico do processo, pois todos esses produtos estão em estoque e, possivelmente, já foram pagos, onerando o custo do almoxarifado da empresa devido à existência de itens que não são utilizados, mas são contabilizados. O fato de a empresa trabalhar com o sistema ETO não justifica o percentual de produtos obsoletos observado, cabendo a este estudo identificar as possíveis causas para talacúmulo de materiais e, então, propor melhorias (ECHEGARAY, 2016).

\subsection{SUGESTÃO DE MELHORIAS}

A fim de identificar as possíveis causas da obsolescência de produtos, realizou-se uma reunião em que foi utilizada a ferramenta brainstorming como apoio para a discussão das ideias relacionadas ao problema principal. No quadro1, expõem-se todas as opiniões e seu setor de origem.

Quadro1 - Quadro de brainstorming

\section{IDEIAS DE POSSÍVEIS CAUSAS}

1. Colocação de compras para projetos especiais sem verificar estoque.

2. Troca de revisão de itens de engenharia sem revisar o que tem em estoque.

3. Substituição de um código que não atende àdata de entrega por outro compatível, PCP após reunião da produção, sem possibilidade de cancelamento da $\mathrm{OC}$ inicial.

4. Cadastro de produtos descentralizados.

\section{ORIGEM}

Comercial/

Compras

Engenharia

Engenharia 
5. Alteração de processo com troca de material, em que, mais vezes, o material que Engenharia sobra é dedicado e/ou específico.

6. Materiais comprados por meio de kits para obtenção de descontos.

Comercial

7. Não se possui um método que informe itens similares no ERP .

Engenharia

Fonte: elaborado pelos autores (2019).

Observa-se que, em projetos de maior complexidade, nos quais o setor comercial necessita do corpo técnico para especificação, a lista de materiais é feita no momento do orçamento ao cliente, o que implica itens, tanto a mais, quanto a menos para o pleno funcionamento conforme o desejado pelo cliente. Essas falhas acontecem, pois, nesse momento, não se tem certeza de todos os dados do projeto; somente após o desenvolvimento do projeto com a estrutura de produto completa, haverá a certeza das quantidades e dos códigos necessários para a fabricação do produto. Assim, é gerada uma pré-lista para orçamento ao cliente, e essa mesma lista é negociada com fornecedores para barganhar preços, desconsiderando os estoques, como também possíveis códigos que estejam parados e que poderiam ser utilizados para substituir a necessidade de compra. Essa causa foi elencada pelo grupo como a mais forte entre as mencionadas.

$\mathrm{O}$ item 2 concerne a itens desenvolvidos internamente, como o chicote elétrico, que passam por constantes revisões. Ao passar por revisões que são originadas pelo cliente, não se tem o procedimento de consumir o estoque, de modo que ele acaba ficando obsoleto e não sendo utilizado. A sugestão para situações como essa seria criar um fluxo para cadastrar o novo produto, passando por uma análise crítica em relação a estoques e a compras, caso fosse revisão de algum outro produto já existente (BOXALL; WINTERTON, 2018).

A necessidade de substituição de produtos, conforme item 3, é originada nas reuniões semanais de PCP, em que se analisam as demandas e se decide, caso algum produto não atenda à data de entrega por motivos como atraso, importação e falta de capacidade do fornecedor, realizar a troca ou substituição desse produto por algum semelhante ou, até mesmo, de outra marca; entretanto, a mercadoria comprada, inicialmente, não é cancelada com o fornecedor. A sugestão, nesse caso, seria adotar o procedimento de cancelar ou trocar o artefato com o fornecedor (SHOU et al., 2018).

O cadastro dos produtos, conforme item 4, também foi evidenciado como uma possível causa de obsolescência, pois existem produtos duplamente cadastrados com referência e códigos iguais. Isso faz que alguns utilizem um código e outros utilizem o código duplo, o que ocorre porque muitos usuários têm acesso livre ao cadastramento de produtos. Para esse caso, a sugestão seria limitar o número de usuários para cadastramento dos produtos (ADETUNJ; BISCHOFF; WILLY, 2018).

As alterações de projeto, constantes no modelo de negócio da empresa (item 5), são igualmente citadas pelos entrevistados. Muitas vezes, o cliente compra a partir do orçamento inicial apresentado, mas, ao desenvolver o projeto, é necessário adicionar ou retirar materiais conforme a necessidade do cliente, o que implica um grande problema para a gestão de materiais, pois se trata de material já comprado e em estoque que, muitas vezes, consiste em artefatos específicos para dada aplicação, ou seja, que não terão utilidade alguma em outros projetos. A sugestão seria enviar esse material excedente ao cliente, a fim de que, mesmo que não seja utilizado, não ficará obsoleto na empresa (NAKANDALA; LAU; SHUM, 2017).

O item 6, por sua vez, evidencia compras estratégicas em forma de kits para conseguir barganhar valores junto ao principal fornecedor. Isso se faz necessário em função das políticas de desconto que o fornecedor oferece como o desconto de $10 \%$ condicionado à compra de um kit em relação ao custo de compra a partir de uma lista de produtos em forma de códigos avulsos. A sugestão para eventos como esse se- 
ria fazer a implosão do kit em itens avulsos para o estoque, para que o ERP e analistas visualizassem todos os componentes como bons para consumo (BOXALL; WINTERTON, 2018).

E oitem 7, por fim, diz respeito ao fato de que, muitas vezes, determinado produto que está em falta conta com um substituto em estoque, mas não é possível saber se são compatíveis por essa informação não constar no ERP. Em face disso, a sugestão seria o ERP disponibilizar, no campo de cadastro, uma aba com os itens similares que poderiam ser utilizados na ausência de algum, ou seja, criar uma tela para facilitar à equipe de desenvolvimento de projetos a utilização máxima dos itens disponíveis em estoque. Tal informação ficaria sob responsabilidade do setor de engenharia (DE MAN; STRANDHAGEN, 2018).

Depois da análise do estudo de caso e proposição das melhorias sugeridas para a empresa, apresenta-se, a seguir, um quadro que sintetiza as ações sugeridas e o possível ganho que a organização teria ao aplicar o método proposto (quadro2). Ressalta-se, ainda, que também seria de grande valia fazer constantes revisões nos procedimentos estabelecidos a fim de garantir a manutenção do sistema para eliminação dos produtos obsoletos, pois, caso contrário, os índices poderão aumentar (JENNINGS, 2018).

Quadro 2 - Ações de melhorias versus impacto

\section{AÇÕES}

\section{IMPACTO}

Gerar pré-estrutura para compra conforme necessidades por meio do MRP, 3\% obsoletos comprando somente o saldo.

Realizar análise crítica de estoques e compras para cadastro de novos produ- $1,5 \%$ obsoletos tos, afim de evitar a duplicidade de códigos e sua obsolescência.

Cancelar compra dos itens substituídos por outros por não atender ao prazo $1 \%$ obsoleto de entrega.

\begin{tabular}{l|l} 
Limitar o número de usuários para cadastro de matéria-prima. & $0,1 \%$ obsoleto
\end{tabular}

Enviar ao cliente materiais que sobraram em decorrência de alteração de pro- Eliminação de jeto solicitada por ele mesmo. itens especiais

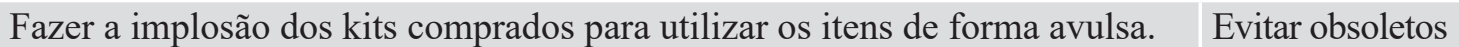
Criar campo similaridade no ERP. Maior utilização

Fonte: elaborado pelos autores (2019).

Com a implementação das ações sugeridas no quadro2, os ganhos seriam refletidos diretamente no indicador de obsolescência, que é medido pela empresa. Embora, atualmente, o foco de trabalho consista na redução dos itens obsoletos, as ações propostas poderiam evitar que os produtos se tornassem obsoletos, o que permitiria, portanto, atacar a raiz do problema (BURNS, 2016).

O percentual de ganho indicado no quadro 2 foi elaborado com dados obtidos do ERP da empresa, podendo variar para mais ou para menos, dependendo do mix de itens. Em relação a algumas ações, não foi possível estabelecer métricas percentuais, pois são práticas que ocasionariam ganhos qualitativos no traba- lho operacional dos analistas e projetistas (DE MAN; STRANDHAGEN, 2018).

A aplicação dessas ações possibilitaria, assim, que o indicador atendesse à meta estabelecida e, além disso, propiciaria uma revisão das metas, visando baixar o índice de produtos obsoletos. Contudo, essa movimentação dos percentuais do indicador somente poderia ser percebida em longo prazo em função da utilização dos componentes que estão na empresa, pois são itens com especificações técnicas por vezes de difícil uso, fazendo que a engenharia de desenvolvimento despenda de um período maior para projetar determinada solução (AQLAN, 2017). 


\section{CONCLUSÕES}

Este artigo identificou as causas de produtos obsoletos e propôs uma nova metodologia para diminuição dos problemas de gerenciamento de estoques para empresas cujo sistema de fabricação seja do tipo ETO. A metodologia aplicada mostrou-se prática, objetiva e eficaz, resultando em procedimentos da gestão de suprimentos de simples e fácil implementação, que afetariam os indicadores de a curacidade e de obsolescência. Esses indicadores constituem instrumentos para a gestão de estoques e para o controle dos itens obsoletos e sua possível aplicabilidade.

Ademais, percebeu-se que a alta direção precisa estar comprometida com as estratégias de abastecimento, colocando-se como incentivadora na aplicação das ações propostas. As barreiras culturais são alguns dos principais problemas na implementação das ações, de modo que a resistência em mudar as formas de trabalho deve ser quebrada, principalmente, por meio de uma definição dada pela direção, focando, primeiramente, o consumo dos itens já obsoletos e, em seguida, a aplicação de ações nas causas que originam a obsolescência.

Pode-se, por meio da abordagem proposta, evitar custos desnecessários com a falta, os excessos e a obsolescência dos produtos em estoque. Conforme constatado, há grandes oportunidades de ganhos na cadeia de abastecimento entre o fornecedor e o cliente com a aplicação do método proposto. Além disso, destaca-se que os custos adicionais provenientes de ações que prejudicam o desempenho das empresas em médio prazo estão sendo pagos pelo consumidor final, o qual, ao perceber essa deficiência, busca produtos alternativos ou procura a concorrência.

Assim, este artigo possibilitou conhecer o processo de fabricação e de controle de estoques de uma indústria do setor elétrico. Nesse sentido, os controles de ERP/MRP, estoques de segurança, produtos com obsolescência programada e indicadores de acuracidade e de obsolescência mostraram a realidade das áreas pesquisadas, evidenciando as causas dos pro- blemas e as propostas de ações para correção.

Ressalta-se, por fim, que o estudo de caso foi realizado em uma empresa de médio porte de abrangência regional, o que impede a generalização dos seus resultados e constitui, portanto, um ponto de limitação da pesquisa. Para investigações futuras, indica-se um estudo quantitativo com profissionais das áreas de gestão da produção e estoques, bem como um estudo qualitativo de múltiplos casos com empresas de segmentos diferentes para um comparativo entre elas.

\section{REFERÊNCIAS}

ADETUNJI, O.; BISCHOFF, J.; WILLY, C. J. Managing system obsolescence via multicriteria decision making. Systems Engineering, v. 21, n. 4, p. 307-321, 2018.

AQLAN, F. Dynamic clustering of inventory parts to enhance warehouse management. European Journal of Industrial Engineering, v. 11, n. 4, p. 469-485, 2017.

BAYRAM, V.; GZARA, F.; ELHEDHLI, S. Joint capacity, inventory, and demand allocation decisions in manufacturing systems. IISE Transactions, v. 51, n. 3, p. 248-265, 2019.

BOXALL, P.; WINTERTON, J. Which conditions foster high-involvement work processes? A synthesis of the literature and agenda for research. Economic and Industrial Democracy, v. 39 , n. 1, p. 27-47, 2018.

BURNS, P. Entrepreneurship and small business. Palgrave: Macmillan Limited, 2016.

CARNIEL-PERRIN, B. et al. Pull-logic and ERP within Engineering-to-Order (ETO): The case of a British Manufacturer. [S.l.: s.n.], 2016.

CASSEL, C.; SYMON, G. Qualitative methods in organizational research: a practical guide. London: Sage, 1994. 
COSTANTINO, F. et al. Spare parts management for irregular demand items. Omega, v. 81, p. $57-66,2018$.

DE MAN, J. C.; STRANDHAGEN, J. O. Spreadsheet Application still dominates Enterprise Resource Planning and Advanced Planning Systems. IFAC-PapersOnLine, v. 51, n. 11, p. 1224-1229, 2018.

ECHEGARAY, F. Consumers' reactions to product obsolescence in emerging markets: the case of Brazil. Journal of Cleaner Production, v. 134, p. 191-203, 2016.

GHOLAMI, A.; MIRZAZADEH, A. An inventory model with controllable lead time and ordering cost, log-normal-distributed demand, and gamma-distributed available capacity. Cogent Business \& Management, v. 5, n. 1, 2018.

HU, Q. Inventory Management and Debt Maturity Structure. Production and Operations Management, v. 26, n. 10, p. 1819-1838, 2017.

JENNINGS, C. P. Forecasting Technical and Functional Obsolescence for Improved Business Processes. 2018. Tese (Doutorado em Psicologia) - Pennsylvania State University, 2018.

LI, M.; WANG, Z. An integrated robust replenishment/production/distribution policy under inventory inaccuracy. International Journal of Production Research, v. 56, n. 12, p. 41154131, 2018.

LIU, X.; WANG, W.; PENG, R. An integrated production, inventory and preventive maintenance model for a multi-product production system. Reliability Engineering \& System Safety, v. 137, p. 76-86, 2015.

NAKANDALA, D.; LAU, H.; SHUM, P. K. C.A lateral transshipment model for perishable inventory management. InternationalJournalofProduction Research, v. 55, n. 18, p. 53415354, 2017.
PESSOTI, H. R.; SOUZA, F. B. Análise dos impactos da migração de um sistema MTS para um sistema ATO nas estratégias de manufatura e competitiva de uma indústria moveleira. In: SIMPÓSIO DE ENGENHARIA DE PRODUÇÃO,12., 2005, Bauru. Anais [...]. Bauru: FEB/UNESP, 2005.

SANDBORN, P. Forecasting technology and part obsolescence. Proceedings of the Institution of Mechanical Engineers, Part B. Journal of Engineering Manufacture, v. 231, n. 13, p. $2251-2260,2017$.

SHOU, Y. et al. Supply chain integration and operational performance: the contingency effects of production systems. Journal of Purchasing and Supply Management, v. 24, n. 4, p. 352-360, 2018.

SILVA, M. A. da. Planejamento colaborativo no gerenciamento de cadeias de suprimentos: um estudo de caso em empresa do setor metal mecânico. [S.l.: s.n.], 2014.

SYLLA, A. et al. How to deal with Engineering-to-Order Product/System Configuration? ConfWS 2018 - 20th Configuration Workshop, Graz, Austria. p. 103-108, 2018.

TEIXEIRA, R. F.; PACHECO, M. E. C. Pesquisa social e a valorização da abordagem qualitativa no curso de administração: a quebra dos paradigmas científicos. Caderno de Pesquisas em Administração, São Paulo, v. 12, n. 1, p. 55-68, 2005.

WANG, Z.; MERSEREAU, A. J. Bayesian Inventory Management with Potential ChangePoints in Demand. Production and Operations Management, v. 26, n. 2, p. 341-359, 2017.

WANKE, P. Gestão de estoques na cadeia de suprimento. São Paulo: Atlas, 2011.

YIN, R. K. Estudo de Caso: planejamento e 
métodos. Porto Alegre: Bookman, 2015.

YLIPÄÄ, T. et al. Identification of maintenance improvement potential using OEE assessment. International Journal of Productivity and Performance Management, v. 66, n. 1, p. 126-143, 2017. 